\title{
The Relation of Helicobacter pylori Infection to Gastric Cancer
}

\author{
Abdulaziz Radhi S ALjohni ${ }^{1,2 *}$, Mohamed Morsi M Ahmed ${ }^{1,3}$ and \\ Bassam Oudh Al Johny ${ }^{1}$ \\ ${ }^{1}$ Department of Biological Science, Faculty of Science, King Abdulaziz University, P O \\ Box 80203, Jeddah 21589, Kingdom of Saudi Arabia. \\ ${ }^{2}$ Department of Laboratory at King Fahad Hospital, Medina, Kingdom of Saudi Arabia \\ ${ }^{3}$ Nucleic Acids Research Department, Genetic Engineering, and Biotechnology \\ Research Institute (GEBRI), Mubarak City for Scientific Research and Technology \\ Applications, Alexandria, Egypt \\ *Corresponding Author: Abdulaziz Radhi S ALjohni, Department of Biological \\ Science, Faculty of Science, King Abdulaziz University, P O Box 80203, Jeddah \\ 21589, Kingdom of Saudi Arabia and Department of Laboratory at King Fahad \\ Hospital, Medina, Kingdom of Saudi Arabia.
}

\begin{abstract}
Background: Helicobacter pylori (H.P.) is a very common bacteria that affect half of the population. It has been incriminated in cancer stomach and gastric lymphoma.

Objective: This work aims to discuss the relation and mechanism of H.P. in causing gastric carcinoma.

Review of Literature: H.P. has different methods of transmission like oral-oral, fecal-oral, gastro-oral and gastro gastric. The microbiological features of H.P. support its growth and protect it from oxidative damage that enhancing its survival rate. H.P. is now determined as one of the most critical carcinogenic infectious agents because it's a high risk of developing stomach cancer. Gastric cancer is the third type of cancer leading to death and it is due to different causes and risk factors. There are many factors affect the outcome of patients who had infected with H.P. and increase the risk of developing cancer. These are environmental, host-related and virulence factors. All of these factors participate in the development of gastric cancer by two mechanisms, direct inflammatory effects and indirect through mutations in the cell-cycle regulating genes.

Conclusion: H.P. infection has an important carcinogenic role in the development of cancer stomach. Eradication of H.P. will decrease the risk of developing stomach cancer.

Keywords: Helicobacter pylori; Gastric Cancer; Transmission; Infection; Relation
\end{abstract}

\section{Abbreviation \\ H.P.: Helicobacter pylori}

\section{Introduction}

Helicobacter pylori (H.P.) is one of the most widespread bacterial pathogens in the world. Over half of the world population is colonized with this bacterium [1]. In Saudi Arabia found that 49\% of Saudi children have an infection in a study made of 303 children [2]. This bacterium can be determined as a microaerophilic bacterium, gram-negative bacillus bacteria that found usually in the stomach. The prevalence rate of infection by this bacterium varies from nation to other [3]. In developing countries with poor socioeconomic conditions and genetic predisposition, the percentage is much higher than that in developed countries [4]. People infected with this bacteria have a major risk factor in developing different gastrointestinal diseases that vary and arranged from chronic gastritis, peptic ulcer and in some cases it can lead to stomach cancer and gastric mucosa-associated with lymphoid tissue lymphoma (MALT) [1]. These diseases are the outcome of the complex interaction of the bacterium and the host [2]. Age is considered as a major risk factor in influence the pathogen, it found that the infec- tion is usually acquired in early childhood [4]. Eighty-five percent of people infected with Helicobacter pylori has no symptoms or complication until it reaches a high level of development [5]. About $15 \%$ of those infected with H.P. will get sick and have symptoms or die due to the infection [5].

H.P. is one of the most critical carcinogenic infectious agents worldwide [6]. Approximately $60-70 \%$ of stomach cancer cases are associated with H.P. [1,7]. Out of 14 million new cancer cases in 2012, 770,000 cases were due to the carcinogenic effect of H.P. [6]. Gastric cancer is the third cause of cancer mortality and the fifth of the most common cancer worldwide [8]. Its fatality risk rate is $75 \%$ [8]. According to global, 2012, there were about (952000) new cases in the world accounting for $6.8 \%$ of all cancer cases and $8.8 \%$ of the total deaths from cancer in both sexes Almost 723,000 death was due to gastric cancer [8]. According to world health organization in 2015, 8.8 million death was due to cancer and the stomach cancer was the $4^{\text {th }}$ most common cancer-causing death 754000 [8]. In kingdom Saudi Arabia according to the Saudi Cancer Registry in 2010, a total of 291 cases of stomach cancer were diagnosed and was ranked the eleventh type of cancer in both male and female [9]. There is gradually increasing in the incidence because of ag- 
ing, growth of the world population and increase of cancer-cause behavior like bad lifestyle and smoking $[8,9]$.

\section{Objectives of the Study}

This review aims to give a brief description of H.P. and gastric cancer also it will focus on the relation between them different factor and how the Helicobacter pylori will lead to stomach cancer.

History and epidemiology of H.P. and gastric cancer

German scientists found bacteria in 1875 lining the human stomach with spiral-shaped but they couldn't culture them, and they didn't find a result. However, after that, the H.P. is discovered in a patient stomach that had gastritis and ulcer in Australia, 1982 [10].

H.P. is one of the most universal infection and has strong variation across the world [11]. Many different factors cause this variation like age of transmission, method of transmission, race, socioeconomic status and Geographic area [4,12]. These factors affect the association between H.P. and the rate of developing stomach cancer in different areas [13]. In Africa, the prevalence of people infected with H.P. is very high, $91 \%$ of the population, but on the other hand, there is a low prevalence of developing stomach cancer $[8,13]$.

\section{H.P. transmission}

According to different sources, humans are the only well established "reservoir" of H. pylori, while the role of other mammalians (cat, pig, primates), as sources of infection, is still controversial [14]. There are many ways and methods in the transmission of H.P., which vary and have different rates of risk $[12,15]$. The most popular mode of transmission is from person to person by different methods oral to oral through sharing the saliva or other rote like fecal to oral when drinking water contamination with fecal, gastro-oral and gastro-gastric that might be transmitted by an endoscopic procedure [12,15]. The leading of this concept is when they found H.P. DNA in saliva, feces, and within the plaque of the teeth [12]. One of the major sources of infection is the waterborne transmission that can be found mostly in poor socioeconomic areas that using untreated water is common [12]. Poor hygiene has also a positive effect on increasing the risk of transmission of H.P. [12]. Within the family, if one of the members had the H.P., there is a high suggestion that other members have H.P. especially from the mother to her children $[12,16]$. Knowledge about the method of transmission of $H$. pylori is important to prevent the spreading of infection.

\section{H.P. microbiology}

H. pylori is a Gram-negative, unipolar, multi-flagellate, considered a spiral organism found especially in human gastric mucosa. Also, it has a helix-formed shape around $3 \mu \mathrm{m}$ long with a breadth of around $0.5 \mu \mathrm{m}$. It is microaerophilic that means it requires oxygen $[1,17]$. The H.P. has two divisions according to its lineage, the gastric Helicobacter species and the enterohepatic (non-gastric) Helicobacter species [1]. H.P. contains Catalase that protects and helps in growth and survives bacteria from oxidative damage by reactive oxygen species (ROS) [18]. It additionally contains ureases, which is a protein that catalyzes the hydrolysis of urea into ammonia and carbon dioxide, that consider as a base that tends to increase the $\mathrm{PH}$ and cause neutralization of the stomach acidity, what's more, The increase in the rate of ammonia will affect intercellular tight junction enhancing the permeability and upsetting the gastric mucous film of the stomach mucin change from gel to sol, so the H.P. will swim openly through the mucosa and join to epithelial cell [19].

Factors affecting the outcome of H.P. infection and increase the risk of gastric cancer

Environmental factors

The diet and type of food have been strongly investigated and it found that a food with a high level of salt will act and work with H.P. [13]. Salty food harm the gastric mucosa leading to H.P. and steadiness that increase the susceptibility to tumorigenesis [13]. Also, eating a high amount of pickled food had been established in increasing the risk of H.P. associated Stomach cancer $[13,20]$. It is dubious whether red meat is a risk factor or not. It was found that red meat appeared to expand the danger of stomach cancer without H.P. through harem iron catalyzing the endogenous formation of carcinogenic N-nitroso compounds [13]. Ikeda F., et al. 2009, reported that hyperglycemia or diabetes is a factor that increases the risk of stomach cancer in subjects with high ( $>6.0 \%)$ HbA1c levels [21].

\section{Host factors}

There are normally occurring genetics changes that may not show themselves in a clinical disorder or pathology yet increment the danger of H.P.-related stomach cancer [22]. An example of genetic alterations is genetics polymorphism or single nucleated polymorphism (SNPs) that alter expression in cytokines involved in inflammation that increase a person's risk of stomach cancer associated with H.P. such as IL-1beta, IL-10, and TNF-alpha that control inflammation and decrease acid secretion [23]. The Prevalence of SNPs varies according to ethnicity and this might be an explanation of why there are differences in host reaction according to people's ethnicity [23].

\section{Virulence factors}

H.P. has two main virulence factors, cytotoxin-associated gene $\mathrm{A}$ (cagA) and vacuolating cytotoxin A (vacA) [24]. Prevalence of cagA and VacA seropositivity, respectively was $82.6 \%$ and $73.91 \%$ in GC patients [25]. In Ohau, Blaser., et al. 1995, reported that infection with a cagA-positive $H$. pylori strain in comparison with a cagAnegative strain somewhat increases the risk for the development of gastric cancer [26]. Sequencing of cagA found that it's a part of cag pathogenicity island and cagA can travel and deliver to the host cell from the bacteria through type 4 secretion system (T4SS) [27]. In host epithelial cell cagA act as signaling for the cell by stimulating cell proliferation and inhibiting apoptosis [28,29]. Also, cagA alters the cell polarity to help the H.P. to adhered and turn the apical cell 
surface into a replicative niche [28,30]. Also, it alters the cellular junction and increases pro-inflammation signals [28,31].

H.P. adheres to the epithelium and grows on it. CagA helps H.P. live by altering an epithelial cell that allows it to obtain nutrition across the epithelial barrier [28]. Another effect of cagA that it increases iron uptake by the epithelium that allows H.P. to grow in low iron conditions [28].

One of the most important mechanisms of H.P. that it blocks anti-microbial peptide secretion from the microphages of epithelial cells by releasing catalase [32]. In another hand, H.P. can also induce macrophage apoptosis by induces NO synthase (iNOS) in macrophages and release of NO that derived from it and this will lead to activating macrophages apoptosis [27,33]. In the end, all these mechanisms will lead to impaired in both adaptive and innate immune responses allowing H.P. to survive [28,33,34]. The other virulence factor vacA also exerts different effects on the target cells, including disruption of mitochondrial functions, stimulation of apoptosis and blockade of T-cell proliferation [35].

Causes and risk factor of stomach cancer

H.P. is a primary carcinogen because long term carrying of H.P. has been found to increase the risk of developing gastric adenocarcinoma approximately 1 to 3 times [36]. In Japan, with a mean follow up 7.8 years report that gastric cancer developed in 36 (2.9\%) inpatient who had H.P. in a prospective study made on 1526 Japanese, 1246 had the Helicobacter pylori and 280 didn't [37]. also found that there is an increase in the risk of who had gastric ulcer to develop gastric cancer about 5 (2.2\%) of 297 patients with gastric ulcer and none of the 275 with duodenal ulcers [37]. Other risk factors include gender its more common in male than female, age it occurs often in age over 40 years old, geographic factor for example Asian individual and south American have a high risk of gastric cancer, Smoking, salt intake, drinking alcohol and its risk effect can be only due high consumptions, Red meat, high fat, Obesity, radiation and family history [38].

\section{Prognosis of stomach cancer}

Prognosis of stomach cancer can be different from patient to another according to different factor and this factor will determine either cancer can be treated or not. The site of the tumor if it's in the lower part of the stomach it can be treated more than in the upper part of the stomach. Another factor is the distance that the tumor invades the stomach wall and if the lymph node is involved or not [39]. If the tumor spread and reach the different area outside the stomach its cant be treated, and the possible treatment is to decrease the symptoms of cancer [39].

\section{Eradication of H.P. decreasing the risk of stomach cancer}

In a study made by, Ma., et al. 2012, with a mean follow up 15 years reported that during nearly 15 years period of treatment by amoxicillin and omeprazole there is a significant decrease in stomach cancer incidence by (39\%) [40]. Also, a mixture of vitamin C, vitamin $\mathrm{E}$ and selenium was significantly causing a reduction in mortality rate from stomach cancer [40]. A meta-analysis made by, Fuccio., et al. 2009, to see the effect of H.P. eradication treatment on reducing the risk of stomach cancer, found that eradication treatment of H.P. will reduce the incidence of stomach cancer by (44\%) $[13,41]$. On the other hand, the massive use of eradication treatment may lead to the initiation of antibiotic-resistant strains of $H$. pylori in the overall public, and additionally an over-utilization of medicinal assets [42].

\section{Role of diet in decreasing the risk of H.P.}

People who eat food with high fiber have shown a significant decrease in H.P. infection [13]. Not only high fiber but the diet include lots of vitamin especially antioxidant vitamins like vitamin c, vitamin e, b-carotene, and a-carotene can be protective of the damage of infection [43]. In a study made by Li., et al. 2014, found that treating of H.P. by antibiotic lead to treat or decrease the risk of stomach cancer [44].

\section{Mechanism of gastric cancer production by H.P.}

H.P. can lead to the development of gastric cancer by 2 mechanisms-indirect and direct.

\section{Indirect mechanism}

It occurs as H.P. induce an inflammation process that will lead to replacing the old cell with a new one through mitosis. After a time, there will be an accumulation of mitotic error this process was explained by Correa's hypothesis (Figure 1) [45]. The presence of the inflammation will lead to atrophic gastritis that will increase the $\mathrm{pH}$ and cause the environment to be alkaline [46]. The alkaline environment will help the H.P. to colonize and start proliferation [46]. Also, gastritis in the antrum cause hyperchlorhydria that will lead to a duodenal ulcer which considers as a risk for developing gastric cancer [46]. The gastritis is caused predominantly by cd4 th1-cell response also neutrophil and macrophages are present

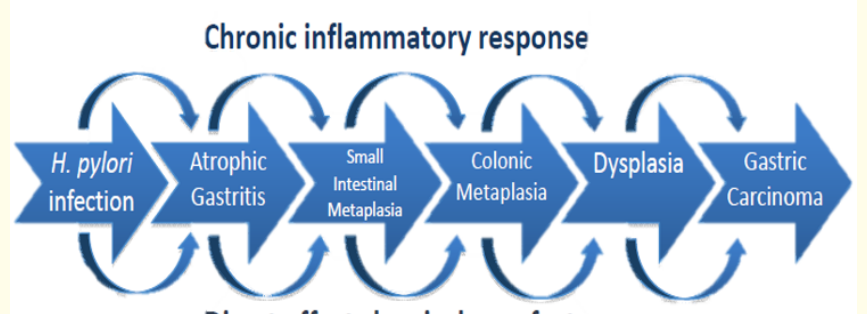

Direct effects by virulence factors

Figure 1: Correa's hypothesis - the histopathologic stages from normal gastric mucosa to gastric carcinoma [13].

which produce reactive oxygen and reactive nitrogen species (ROS/ RNS) [37]. These all combine with hydroxyl ion will lead to oxidative stress then DNA damage [37].

\section{Direct mechanism}

The H.P. influences the gastric epithelial cells by the toxic action of its virulence factors and this will lead to mutations in the cellcycle regulating genes, inadequacies DNA repair, loss of the adhe- 
in-associated gene A ( $\operatorname{cag} \mathrm{A})$ and vacuolating cytotoxin A (vacA) that shown through extensive research to be associated with gastric lesion and its progression to be malignant [48]. CagA positive found to cause by producing an inflammatory reaction a progression from gastritis to atrophy of the glandular mucosal cells and a higher risk of GC [49]. Then cagA enters the epithelial cell with peptidoglycan through a bacterial type IV secretion system and it's encoded by the campaign [49]. CagA will induce different cellular signals such as mitogen-activated protein kinase (MAPK [49]. Peptidoglycan will induce NF- $\kappa \mathrm{B}$ expression and phosphoinositide-3 kinase (PI3K-AKT) signaling pathways [49]. Also, cagPAI+ will enhance the expression of an enzyme called Activation Induced Deaminase (AID) in the gastric mucosa that will lead to mutation in the DNA [50]. VacA participates in enhancing the longevity of the H.P. by disrupting the epithelial cell barrier and suppressing the T-cell response. Also, it disrupts the proliferation of apoptosis balance by the activation of proinflammatory signal pathways that targets the mitochondria and causes programmed cell death [51]. A combination of all these factors affect will lead to gastric cancer [46].

\section{Conclusion}

H.P. infection has an important carcinogenic role in the development of cancer stomach. Eradication of H.P. will decrease the risk of developing stomach cancer. Gastric cancer is a major leader of death with a high fatality rate and there is a gradual increase in the incidence due to some factors. There is a strong relation between H.P. and Gastric cancer that can be due to different factors including environmental, host and virulence factors. On the other hand, you can't just say that the only cause of gastric cancer is the H.P. pylori because there are other causes and risk factors. But H.P. is considered now as one of the major causes that should be focused on when we want to treat cancer because eradication of H.P. has a strong effect on stomach cancer.

\section{Bibliography}

1. Kusters J., et al. "Pathogenesis of Helicobacter pylori Infection”. Clinical Microbiology Reviews 19.3 (2006): 449-490.

2. Hasosah M., et al. "Prevalence and risk factors of Helicobacter pylori infection in Saudi children" a three-year prospective controlled study". Helicobacter 20.1 (2015): 56-63.

3. McColl KEL. "Helicobacter pylori infection". The New England Journal of Medicine 362.17 (2010): 1597-1604.

4. Hunt RH e. "Helicobacter pylori in developing countries. World Gastroenterology Organization Global Guideline". Journal of Gastrointestinal and Liver Diseases 20.3 (2011): 299-304.

5. Bytzer P., et al. “Diagnosis and treatment of Helicobacter pylori infection”. Danish Medical Bulletin 58.4 (2011): C4271.

6. Plummer M. "Global burden of cancers attributable to infections in 2012: a synthetic analysis". The Lancet Global Health's 4.9 (2016): e609-e616.
7. Chang, AH and Parsonnet J. "Role of Bacteria in Oncogenesis". Clinical Microbiology Reviews 23.4 (2010): 837-857.

8. Fock KM. "Review article: the epidemiology and prevention of gastric cancer". Alimentary Pharmacology and Therapeutics 40 (2014): 250-260.

9. "Saudi Cancer Registry Cancer Incidence Report in Saudi Arabia 2010". Riyadh (KSA): Saudi Cancer Registry 16 (2014): 5456.

10. Watts G. "Nobel prize is awarded to doctors who discovered $\mathrm{H}$ pylori”. British Medical Journal 331.7520 (2005): 795.

11. JE E. "Recent developments in the epidemiology of Helicobacter pylori". Gastroenterology Clinics of North America 29.3 (2000): 559-578.

12. LM Brown. "Helicobacter pylori: epidemiology and routes of transmission". Epidemiologic Reviews 22.2 (2000): 283-297.

13. Sauid Ishaq L. "Helicobacter pylori and gastric cancer: a stateof-the-art review. PubMed Central (PMC)". Gastroenterology and Hepatology from Bed to Bench 8.1 (2015): S6-S14.

14. Tursi A., et al. "[The modes of transmission of Helicobacter pylori infection]". Recenti Progressi in Medicina 88.5 (1997): 232-236.

15. DR Cave. "How is Helicobacter pylori transmitted?" Gastroenterology 113.6 (1997): S9-S14.

16. Y Kivi. "Helicobacter pylori occurrence and transmission: a family affair?" Scandinavian Journal of Infectious Diseases 38.67(2006): 407-417.

17. M Hill. "Microbiology of Helicobacter pylori". Gastroenterology Clinics of North America 22.1 (1993): 5-19.

18. Hazell SL., et al. "Helicobacter pylori catalase". Journal of General Microbiology 137.1 (1991): 57-61.

19. HL Mobley R. "Microbial ureases: significance, regulation, and molecular characterization". Microbiological Reviews 53.1 (1989): 85-108.

20. El-Omar EM., et al. "Interleukin-1 polymorphisms associated with increased risk of gastric cancer". Nature 404 (2000): 398402 .

21. Ikeda F., et al. "Hyperglycemia increases risk of gastric cancer posed by Helicobacter pylori infection: A population-based cohort study". Gastroenterology136 (2009): 1234-1241.

22. Honarmand-Jahromy S. "Reciprocal impact of host factors and Helicobacter pylori genotypes on gastric diseases". World Journal of Gastroenterology 21.31 (2015): 9317-9327. 
23. Figueiredo C. “Cytokines, cytokine gene polymorphisms and Helicobacter pylori infection: Friend or foe?" World Journal of Gastroenterology 20.18 (2014): 5235-5243.

24. Yamaoka Y. "Mechanisms of disease: Helicobacter pylori virulence factors". World Journal of Gastroenterology Nature Reviews Gastroenterology and Hepatology 7.11 (2010): 629-641.

25. Renzo Suriani M. “CagA and VacA Helicobacter pylori antibodies in gastric cancer". Canadian Journal of Gastroenterology and Hepatology 22.3 (2008): 255-258.

26. Blaser MJ e. "Infection with Helicobacter pylori strains possessing cagA is associated with an increased risk of developing adenocarcinoma of the stomach". Cancer Research 55.10 (1995): 2111-2115.

27. Yong X., et al. "Helicobacter pylori virulence factor CagA promotes tumorigenesis of gastric cancer via multiple signaling pathways". Cell Communication and Signaling 13 (2015): 30.

28. Tan S., et al. "Helicobacter pylori Perturbs Iron Trafficking in the Epithelium to Grow on the Cell Surface". PLOS Pathogens 7.5 (2011): e1002050.

29. Neal J., et al. "H. pylori virulence factor CagA increases intestinal cell proliferation by Wnt pathway activation in a transgenic zebrafish model". Disease Models and Mechanisms 2013 6 (2013): 802-810.

30. Tan S., et al. "Helicobacter pylori Usurps Cell Polarity to Turn the Cell Surface into a Replicative Niche". PLOS Pathogens 5.5 (2009): e1000407.

31. Bronte-Tinkew D., et al. "Helicobacter pylori Cytotoxin-Associated Gene A Activates the Signal Transducer and Activator of Transcription 3 Pathway In vitro and In vivo". Cancer Research 69.2 (2009): 632-639.

32. Algood H and Cover T. "Helicobacter pylori Persistence: An Overview of Interactions between H. pylori and Host Immune Defenses”. Clinical Microbiology Reviews 19.4 (2006): $597-$ 613.

33. Chaturvedi R e. "Induction of polyamine oxidase 1 by Helicobacter pylori causes macrophage apoptosis by hydrogen peroxide release and mitochondrial membrane depolarization". Journal of Biological Chemistry 279.38 (2004): 40161-40173.

34. Gobert AP e. "Helicobacter pylori induces macrophage apoptosis by activation of arginase II". Journal of Immunology 168.9 (2002): 4692-4700.

35. Palframan S., et al. "Vacuolating cytotoxin A (VacA), a key toxin for Helicobacter pylori pathogenesis". Frontiers in Cellular and Infection Microbiology 2 (2012): 92.

36. Wroblewski L., et al. "Helicobacter pylori and Gastric Cancer: Factors That Modulate Disease Risk". Clinical Microbiology Reviews 23.4 (2010): 713-739.
37. Uemura N., et al. "Helicobacter pylori infection and the development of gastric cancer". The New England Journal of Medicine 345 (2001): 784-789.

38. Lee YY and Derakhshan MH. "Environmental and lifestyle risk factors of gastric cancer". Archives of Iranian Medicine 16.6 (2013): 358-365.

39. Hakimeh Zali M., et al. "Gastric cancer: prevention, risk factors and treatment". Gastroenterology and Hepatology from Bed to Bench 4.4 (2011): 175-185.

40. Ma J., et al. "Fifteen-Year Effects of Helicobacter pylori, Garlic, and Vitamin Treatments on Gastric Cancer Incidence and Mortality". Journal of the National Cancer Institute 104.6 (2012): 488-492.

41. Fuccio L., et al. "Meta-analysis: can Helicobacter pylori eradication treatment reduce the risk for gastric cancer?" Annals of Internal Medicine 151.2 (2009): 121-128.

42. Bin Lu and Meng Li. "Helicobacter pylori eradication for preventing gastric cancer". World Journal of Gastroenterology 20.19 (2014): 5660-5665.

43. Li P., et al. "Association between dietary antioxidant vitamins intake/blood level and risk of gastric cancer". International Journal of Cancer 135.6 (2014): 1444-1453.

44. Li W., et al. "Effects of Helicobacter pylori Treatment on Gastric Cancer Incidence and Mortality in Subgroups". Journal of the National Cancer Institute 106.7 (2014): 116.

45. Correa P. "Human gastric carcinogenesis: A multistep and multifactorial process - First American Cancer Society Award lecture on cancer epidemiology and prevention". Cancer Research 52 (1992): 6735-6740.

46. Correa P., et al. "A model for gastric cancer epidemiology". Lancet 2 (1975): 58-59.

47. Bartchewsky W Jr., et al. "Effect of Helicobacter pylori infection on IL-8, IL-1beta and COX-2 expression in patients with chronic gastritis and gastric cancer". Scandinavian Journal of Gastroenterology 44 (2009): 153-161.

48. Niwa T., et al. "Inflammatory processes triggered by Helicobacter pylori infection cause aberrant DNA methylation in gastric epithelial cells". Cancer Research 70 (2010): 1430-1440.

49. Baghaei K., et al. "Determination of Helicobacter pylori virulence by analysis of the cag pathogenicity island isolated from Iranian patients". Digestive and Liver Disease 41 (2009): 634638.

50. Gonzalez CA., et al. "Helicobacter pylori cagA and vacA genotypes as predictors of progression of gastric preneoplastic lesions: A longterm follow-up in a high-risk area in Spain". The American Journal of Gastroenterology 106 (2011): 867-874. 
51. Huang JQ., et al. "Meta-Analysis of the relationship between cagA seropositivity and gastric cancer". Gastroenterology 125 (2003): 1636-1644.

Assets from publication with us

- Prompt Acknowledgement after receiving the article

- Thorough Double blinded peer review

- Rapid Publication

- Issue of Publication Certificate

- High visibility of your Published work

Website: https://www.actascientific.com/

Submit Article: https://www.actascientific.com/submission.php Email us: editor@actascientific.com

Contact us: +919182824667 